\title{
Investigating the bioavailability of anthocyanin metabolites
}

\author{
R. de Ferrars ${ }^{1}$, A. Cassidy ${ }^{1}$, P. Curtis ${ }^{1}$, C. Czank ${ }^{1}$, Q. Zhang ${ }^{2}$, K. Kalowole ${ }^{2}$, \\ N. Botting ${ }^{2}$ and C. D. Kay \\ ${ }^{1}$ Department of Nutrition, Norwich Medical School, University of East Anglia, Norwich NR4 7TJ and \\ ${ }^{2}$ School of Chemistry, University of St Andrews, KY1 69AJ, UK
}

Anthocyanins (ACN) are a sub-class of flavonoids, found within many red berries and vegetables and have been linked to a decrease in cardiovascular disease (CVD) risk factors ${ }^{(1)}$. However, bioavailability studies have consistently reported extremely low recoveries $(<0.1 \%$ of administered dose) within biological fluids. ACN rapidly degrade into their phenolic acid and aldehyde constituents at neutral $\mathrm{pH}^{2}$ ) and may be further metabolised, forming many possible methyl, glucuronide, sulfate and glutathione conjugates. This work focuses on identifying these unknown ACN metabolites.

Previously published solid phase extraction (SPE) methods ${ }^{(2)}$ were optimised to obtain high extraction efficiencies for an extensive range $(n=51)$ of putative metabolites in urine $(84.4 \% \pm 19.4)$ and serum $(84.5 \% \pm 15.9)$ samples. High performance liquid chromatography (HPLC-UVvis) and mass spectrometry (MS) conditions were also optimised.

Clinical samples from a 12-week anthocyanin intervention where 52 postmenopausal females were fed $500 \mathrm{mg} /$ day elderberry extract ${ }^{(3)}$ were analysed for the presence of anthocyanin metabolites. To date, we have identified nine metabolites (Fig. 1). In addition, post bolus samples indicate an increased excretion of a number of currently unidentified metabolites relative to baseline values (data not shown), which are the focus of future investigation.
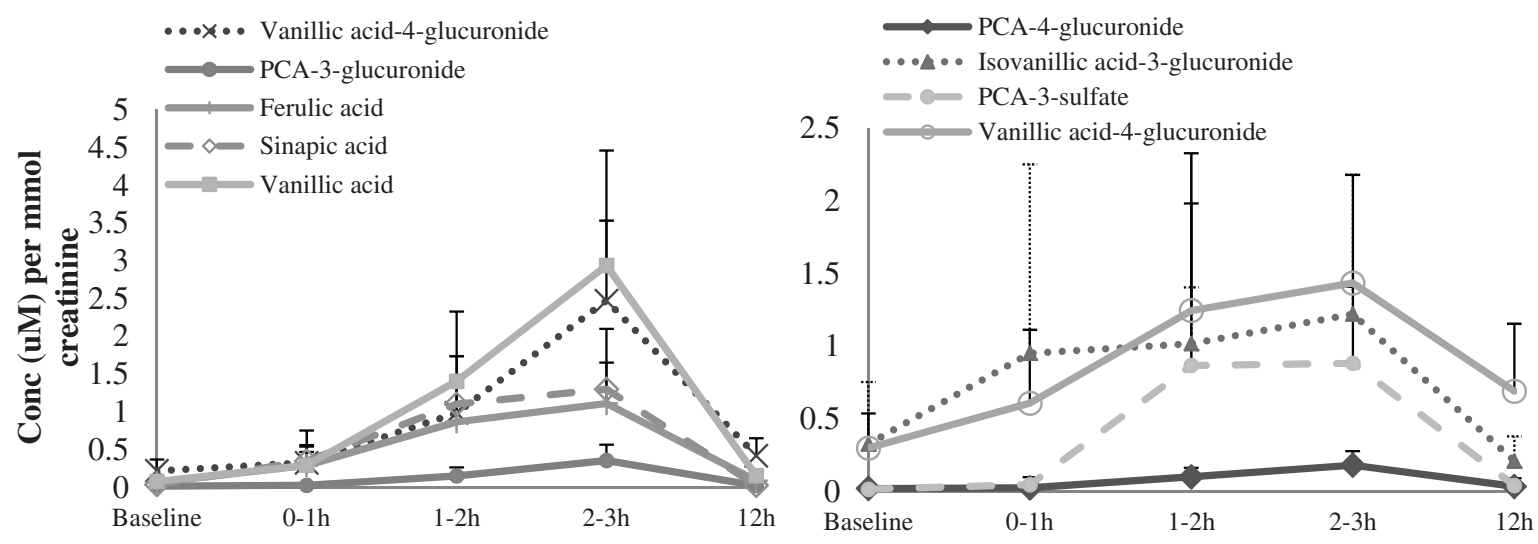

Figure 1: Excretion of anthocyanin metabolites in urine $0-12 \mathrm{~h}$ post bolus $(n=8)$.

The development of these analytical methods has allowed the identification of previously unknown ACN metabolites. These results indicate that ACN degrade and are further extensively metabolised in vivo. The origin of these metabolites will be confirmed through the analysis of samples derived from a recent ${ }^{13} \mathrm{C}_{5}$-labelled cyanidin-glucoside feeding study $(500 \mathrm{mg}$ bolus; $n=8)$. Identifying the metabolic fate of ACN is a vital step towards fully understanding their bioactive properties. Further work into bioactivity of these metabolites may ultimately result in the development of refined dietary recommendations for the prevention of CVD.

1. Cassidy A, O'Reilly ÉJ, Kay C, et al. (2011) Am J Clin Nutr 93, 338-47.

2. Woodward G, Kroon P, Cassidy A, et al. (2009) J Agr Food Chem 57, 5271-8.

3. Curtis PJ, Kroon PA, Hollands WJ, et al. (2009) J Nutr 139, 2266-71. 